370. Sonderausstellung

des Wien Museums

11. März bis 21. August 2011

Eine Ausstellung in Kooperation mit dem Kupferstichkabinett der Akademie der

St. Stephan / An exhibition in colllaboration

with the Grophic Collection of the Academy

of Fine Arts Vienna and the Cathedral Lodge

at St. Stephen's

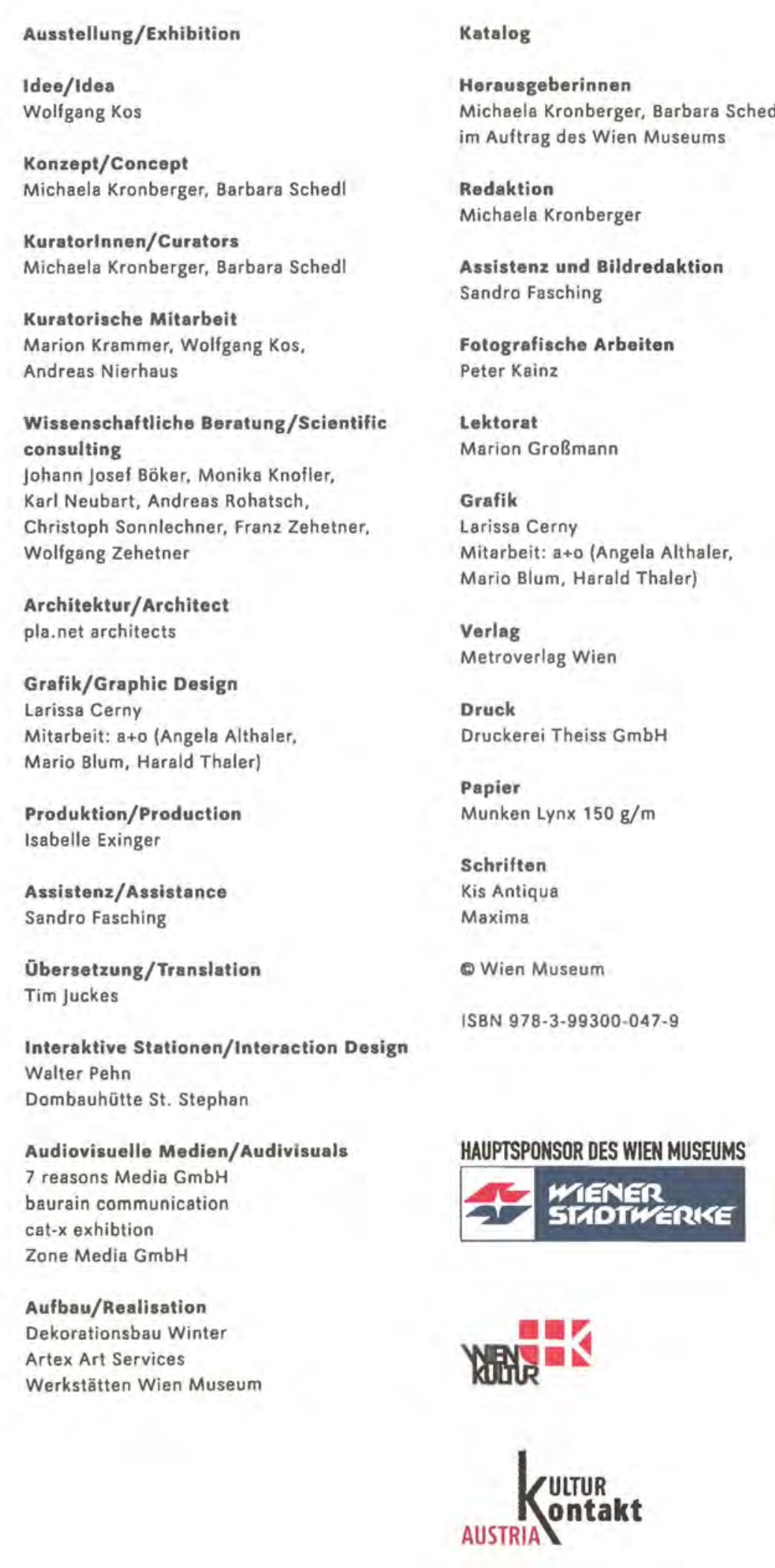

DER DOMBAU VON ST. STEPHAN DIE ORIGINALPLÄNE AUS DEM MITTELALTER 
Wolfgang Kos

Vorwort

Seite 6

Michaela Kronberger und Barbara Schedl

Einleitung

Seite 8

Aufsätze

Monika Knofler

Von der Dombauhütte in die

Sammlungen

Die Odysee der gotiscben Planrisse

Seite 22

Barbara Schedl

Der beschwerliche Weg zum Dom Die Baugescbicbte von St. Stephan

Seite 28

Wolfgang Zehetner

Wissenszentrum und Werkstätte

Die Gescbicble der Wiener Dombaubütte

Seite 36

Johann Josef Böker

Die Schöpfer der Planrisse

Die Baumeister von St. Stephan

Seite 44

Andreas Rohatsch

... Hie sind vermerkt die fertt von Au und von Menestorf ...

Die Steinbrickbe

Seite 50
Michael Viktor Schwarz

Hoch hinaus

Gotische Türme im Wettbewerb

Seite 54

Karl Neubarth und Rudolf Koch

Vom Entwurf zum Werkstein

Mittelalterlicher Baubetrieb zwiscben Tradition und Innovatio

Seite 68

Michaela Kronberger

... hincz sand Stephan zu dem pau ...

Zur Finanzierung des Kirchenbautes von St. Stephan

Seite 74

Barbara Schedl

Eine Kirche bauen - eine Kirche nutzen

St. Stephan im mittelalterlichen Gebraucb

Seite 80

Eva-Maria Orosz

Auf den Spuren des farbigen Lichts

Die Glasfenster von St. Stepban

Seite 90

Christoph Sonnlechner

Kirchenbau und Umwelt

Eine ökologische Kontextualisierun

Seite 96

Andreas Nierhaus

Vollendung unerwünscht

Zur Restaurierung von St, Stepban im 19, fabrbunder

Seite 100

Wolfgang Kos

Erkennbarkeit garantiert

Bildgescbicbte des Stephansdomes von 1500 bis beute

Seite 112
Katalog

Die Geschichte der Planrisse

Seite 122

Gotische Planrisse

Seite 124

3

Entwerfen im Mittelalter

Seite 126

Vom Entwurf zum Werkstück

Seite 132

Vielschichtige Konstruktionen Seite 136

Maßgetreu

Seite 138

7

Vielseitige Kirchennutzung

Seite 142

St. Stephan und seine Türme

Seite 148

Die Dombauhütte zu Wien Seite 156
Die Bedeutung der Baumeiste

Seite 164

Baumaterial für die Stephanskirche Seite 168

Die Großbaustelle im Herzen der Stadt Seite 170

Lebensbedingungen im Spätmittelalter Seite 172

Monster und allerlei Getier

Seite 176

Die Finanzierung von St. Stephan Seite 180

Restaurieren oder Weiterbauen Seite 186

Die Zerstörung des Domes 1945

Seite 192

Anhang

Literaturverzeichnis Seite 198

Leihgeber und Abbildungsnachweis Seite 203

Autorinnen und Autoren Seite 204

Dank Seite 205

Glossar Seite 206 


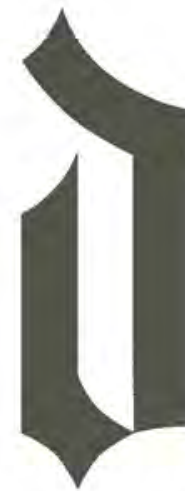

bislang überzeugend dargestellte Baugeschichte der über Jahrhunderte archäologische Forschungen alles andere als gesichert $(A b b$. 1). Die Anfänge und das Aussehen der Stephanskirche im 12. Jahrhundert sind kaum zu rekonstruieren, denn zu ungenau sind die Formulierungen in den Schriftquellen, zu komplex sind die Grabungen, die in den letzten Jahren durchgeqührt fuhrt älteste erhaltene Teil der Stephanskirche anzusehen ist. Überprüft man die Aussagen der Schriftquellen des 12. Jahrhunderts, so findet man keinerlei Hinweise, die eindeutig auf St. Stephan bezogen werden können:

Der Transaktionsvertrag, der so genannte Mautener

Tauschvertrag aus dem Jahre 1137 zwischen dem Babenberger Markgraf Leopold und dem Passauer Bischof Reginmar, wird gerne als die Geburtsstunde der Stephanskirche gesehen. Er besagt aber lediglich, dass die Peterskirche in Wien - damals die Wiener Pfarrkirche - und der dazugehörige Grundbesitz, dem Passauer Bischof übergeben wurden. Dafür erhielt der Baben-
Barbara Schedl

Der beschwerliche

Weg zum Dom

Die Baugeschicbte von St. Stephan erger einen Weingarten bei Wartberg und

besitz, der aber in der Urkunde nicht näher beschrieben ist. Auch eine für das Jahr 1147 quellenmäßig überlieferte Kirchenweihe, die der Passauer Bischof in Anwesenheit des Pfarrers von Wien vollzog, bezieht sich nicht ausdrücklich auf die Stephanskirche.

Die archäologischen Untersuchungen, die in den letzten Jahren im Westbereich und im Langhaus der heutigen Kirche erfolgten, sind äußerst komplex und vieldeutig interpretierbar: Die ältesten Befunde weisen auf eine wohl römische Besiedelung in der ehemaligen Lagervorstadt, also östlich des Legionslagers Vindobona, hin. Über dieser Grabungsschicht fand man Bestattungen, die vermuten lassen, dass diese zu einer nicht weiter definierbaren (kleineren) Kirche gehört haben, die weiter östlich des heutigen Baues bzw. des Riesentors gelegen war. Unsicher ist auch die Darstellung der Bodenuntersuchungen, die in der Nachkriegszeit (1945) getätigt wurden: Damals konnten die Ostteile des vermutlich im 12.Jahrhundert errichteten Baues freigelegt werden, die ein Querschiff erkennen ließen, dessen Ausdehnung die Dimension des hochgotischen Chores vorgab. Allerdings ging die wissenschafliche Dokumentation dieser Grabung verloren; sie wurde aus dem Gedächtnis der damals Beteiligten rekonstruiert.

Alles in allem lässt sich für die Frühzeit festhalten, dass es vor der Wiener Stadtmauer des 12. Jahrhunderts wohl eine Kirche gab. (Abb.2)Wie diese aussah, welche Dimensionen sie hatte, auf leibt indes ein Westturmpaar hatte, dessen aufgehendes Mauerwerk in den unteren Geschossen der heutigen Heidentürme erhalten geblieben ist.

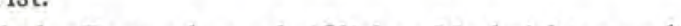
architektonisch Fassbarem: Am 30. März 1220 stellte der Babenberger-Herzog Leopold VI. (1198 -1230) eine Urkunde zugunsten des Schottenklosters in der Stephanskirche aus. Bei diesem Rechtsakt war eine hochkarätige Klientel anwesend, wie aus der mit unterzeichneten Zeugenreihe ersichtlich ist. Es kann also mit Recht geschlossen werden, dass sich die Stephanskirche damals zumindest in einem teilweise benutzbaren Zustand befand. Wann kirche, Situation um 1360 or der Stadt, Situation des
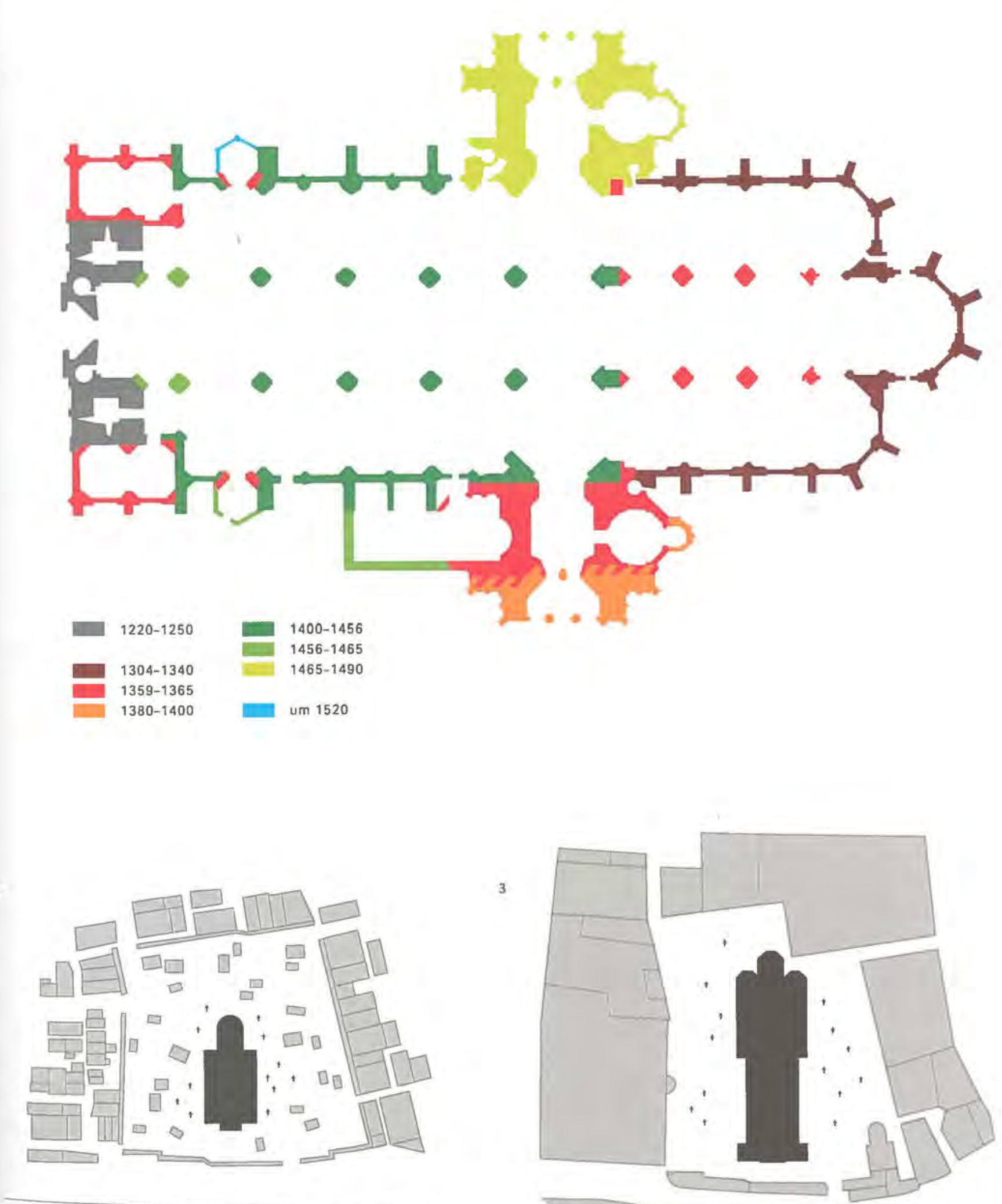

arstellung der Stephanskircho

Darstellung der Stephans.

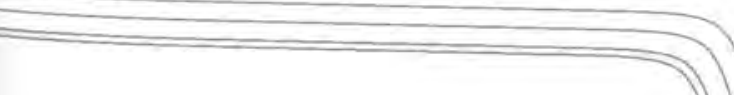

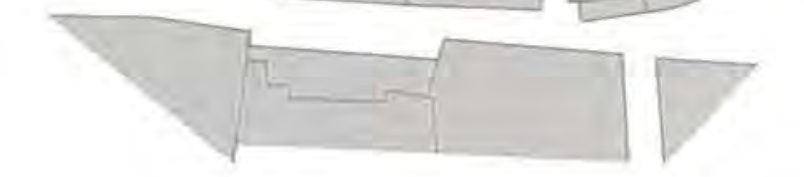


Zu vermuten ist, dass im Zuge der Neukonzeption der Wiener Stadtmauer (ab 1195) unter dem Babenberger Leopold V. (1157-1194) und vor allem unter den ehrgeizigen städtepolitischen Maßnahmen Herzog Leopolds VI. (1176-1230) für die Stadt Wien - zu erinnern ist an die Stadtrechtsprivilegien von 1208 und 1221 - auch anspruchsvolle Bauproekte initiert wurden. Mit der Anlage der neuen Stadtmauer war Wien um das Dreifache . er Pfarrkirche St. Stephan großzügig ausbauen zu wollen. Wer allerdings der Initiator des Bauvorhabens war - der Landesfürst, der Bischof von Passau, zu dessen Bistum Österreic damals zählte, oder die Wiener Pfarrgemeinde - bleibt ungewiss.

Im Wesentlichen ist der Weston Toranlage mit dem dekorativen Portaltrichter (Riesentor) und Teile der Westempore des heutigen Domes nach stilistisch (eindeutigem) Befund dieser frühgotischen Bauphase zuzuordnen, allerdings gab es auch mehrere Planwechsel. Die bedeutendste Änderung war die Ausweitung der Empore in die seitlich liegenden Geschosse der Türme wie auch gegen das Kirchenschiff nach Osten $(A b b .5)$. Diese frühgotische Kirche dürfte ein basilikales Langhaus und ein Querschiff besessen haben und glich in ihrer Dimension und Erscheinung am ehesten dem zeitgleich errichteten Dom von Wiener Neustadt. Ein Brand im Jahre 1258 machte Restaurierungsarbeiten notwendig; 1263 konnte die Kirche letztendlich geweiht werden.

Anfang des 14. Jahrhunderts initiierten die Wienerinnen und Wiener einen A

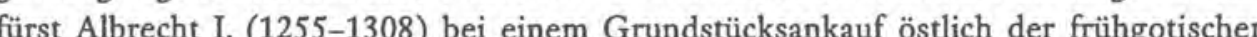
Stephanskirche zwischen dem Kloster Zwettl als Grundstücks-Eigentümer, und den Wienerinnen und Wienern. Mehrere Ablässe $(1323,1326,1327,1339,1340)$ und Stifungen der landesfürstlichen Familie (1330) aber auch der Stadtbevölkerung (1334, 1338 , 1339) weisen auf zunehmende finanzielle Mittel und eine rege Bautätigkeit hin, sodas im Frühjahr 1340 eine Weihe des Chores sowie von sechs Altären stattfinden konnte. Das Bauprojekt dürfte aber damals noch nicht vollendet gewesen sein, denn in den folgenden Jahren wurden weitere Ablassbriefe $(1341,1343)$ ausgestellt $(A b b .3)$.

Bislang war man davon ausgegangen, dass - trotz konstatierter Stilunterschied in der Bauausführung des Chores - dieser Bauabschnitt einer einzigen Bauphase angehör und allein in der Regierungszeit Herzor Albrechts II (1298-1358) entstanden ist und bereits 1340 fertig sestelltwar Eine Bas bering

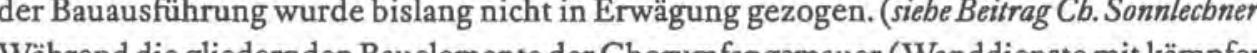
Wahrend die gliedernden Bauelemente der Chorumfangsmauer (Wanddienste mit kämpferlosen Birnstabprofilen), aber auch die Maßwerkformen der Fensterzonen sowie dere Profile äußerst feingliedrig gestaltet sind und dem damaligen (internationalen) Hofst entsprachen, besitzt der Chor-Innenraum massiv ausgebildete Bündelpfeiler mit hohen Sockeln, die sehr blockhaft und kubisch wirken (Abb.4).

Eine derartig massive Bauweise findet sich auch bei dem nördlich und südlich der Heidentürme errichteten Kapellenpaar, die nachweislich mit Herzog Rudolf IV. (1339-1365) - belegt sind Weihedaten in den Jahren 1365 und 1366 - in Verbindung zu bringen sind. Diese, die frühgotische Westfassade umklammernden Kapellenbauten, ware als exklusive - nicht öffentliche - Andachtsräume konzipiert und wurden vom Herzog mit zahlreichen Reliquienschätzen ausgestattet. Nicht nur mit der Errichtung der herzoglichen

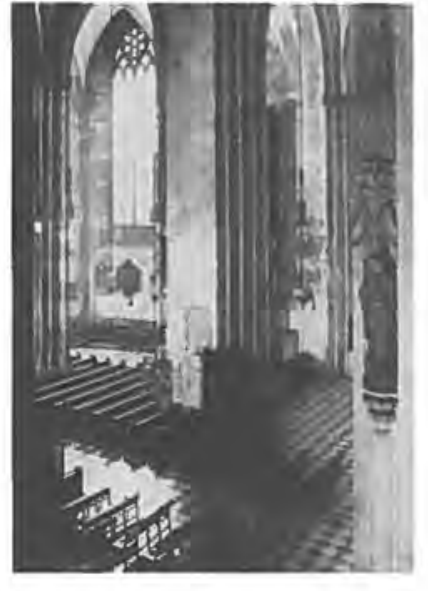

$$
\text { Karl Pani }
$$

Blick vom OrgelfuB in Vierung

und Chor von St. Stephan, 2001

\section{Peter Kodera}

Westfassade von St. stophen,

$1965-1970$
Eotografie

tografie

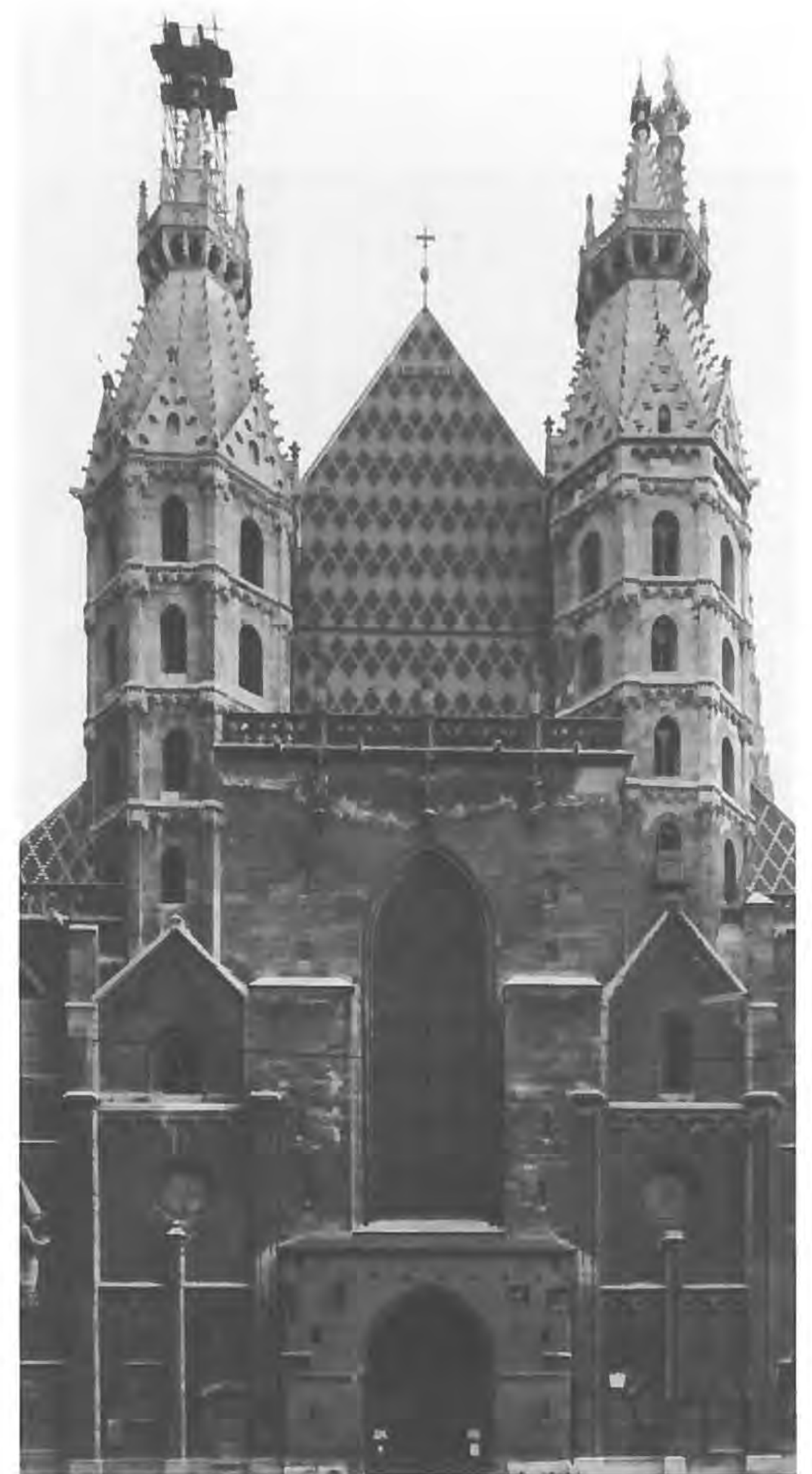


Kapellen im Westen griff Rudolf IV. in das Baukonzept der Wiener Pfarrkirche ein, be- 6 sonders mit der Gründung eines Kollegiatsstiftes und der Errichtung seiner Grablege im Chor änderte sich deren Funktionalität, aber auch das Erscheinungsbild. Bereits im März 1359 erfolgte der erste Spatenstich und im April 1359 die Grundsteinlegung zum Erweiterungsbau von St. Stephan durch den Herzog.

Zahlreiche Ablässe aus den Jahren 1359, 1360,1361,1362,1363,1364 lassen nun wieder auf enorme Einnahmen schließen, die furr den Ausbau des Chores mit der Errichtung der ferzoliche Grablege und ung der herz deutig belegt - bereits auch fur die Verwirklichung des ehrgeizigen Turmprojekts verwendet wurden. Denn erst der Theologe Thomas Ebendorfer $(1388-1465)$ berichtete in der Mitte des 15.Jahrhunderts, RudolfIV. hätte den Grundstein zum Turm gelegt und er wusste weiters von einer Zusammenkunft bedeutender Baumeister, die ihre jeweiligen Projekte vorstellten, zu erzählen, unter denen dann ein Meister von dem Landesfürsten ausgewählt worden sei (siebe Beitrag 7. F. Böker).

Nach Ebendorfers Bericht kam es Anfang des 15.Jahrhunderts (1407) zu einer entscheidenden Planänderung. Dramatisch schilderte er, dass wegen gravierender Abweichungen vom ursprünglichen Turm-Bauplan alles andersartig Errichtete abgetragen werden musste, und zwar bis zu der Stelle, wo der erste Meister das Werk beendet hatte. Die entscheidende Planänderung betraf den Übergang zwischen dem massiven Turmunter-

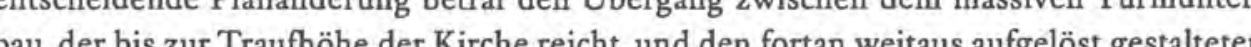
Freigeschossen, die in dem schlanken, zugespitzten Turmhelm gipfeln $(A b b .6)$.

Zur selben Zeit, als der Südturm allmählich emporwuchs, begann man den Neubau eines modernen, größeren Langhauses. Bislang sah man Herzog Rudolf IV. als Initiator des Hallenlanghauses. Die doch sehr einheitliche Ausführung und Konzeption spricht aber für eine kürzere Bauzeit, deren Beginn knapp vor 1400 anzusetzen ist, wie auch zahlreiche Ablässe $(1395,1398,1399)$ und Testamentsstiftungen der Stadtbevölkerung belegen.

Unter Beibehaltung des alten Langhauses wurde zunächst - beginnend beim Turm - die südliche Außenmauer nach Westen zu der südlichen Herzogskapelle vorgezogen; den zweiten Bauabschnitt stellte der von West nach Ost kontinuierlich verlaufende Aufbau der Nordmauer da. Noch bevor man den Anschluss an den 1450 begonnenen Nordturm erreichte, wurde zwischen 1422 und 1430 das frühgotische Langhaus abgetragen. Nach Beseitigung der alten Bauteile konnten dann die neuen Mittelschiffarkaden in (143). Turms vollendet (1433).

In den 40er Jahren des 15. Jahrhunderts kam es beim inneren Ausbau des neuen Langhauses zu einer Planänderung. Der bisherige Entwurf sah eine klassisch-hochgotische Gestaltungsweise vor. Die Seitenschiffe sollten ursprünglich mit fünfteiligen Kreuzrippengewölben ausgeführt werden; der Raumcharakter hätte dem rudolphinischen Chorkonzept entsprochen. Mit der Berufung eines neuen Dombaumeisters, dem vielseits bekannten Hans Puchsbaum im Jahre 1446, erfuhr dieses Konzept eine deutliche Korrektur, indem nun die Mittelschiffarkaden und auch der mittlere Teil der Westwand deutlich erhöht wurden, sodass eine gestaffelte Langhaushalle entstand. Beim Tod von Hans Puchsbaum (kur nach 1454) entsprach die Stephanskirche in ihrer Außengestalt mit Südturm und mit dem charakteristischen hohen Dach, dessen Eindeckung mit den farbigen Ziegeln 1449 begonnen worden war, dem heutigen Erscheinungsbild. Im Inneren allerdings fehlte die Wölbun und im Norden stand ein großer Bereich offen da der Nordturm erst fundamentiert wat:

\section{Peter Kodera}

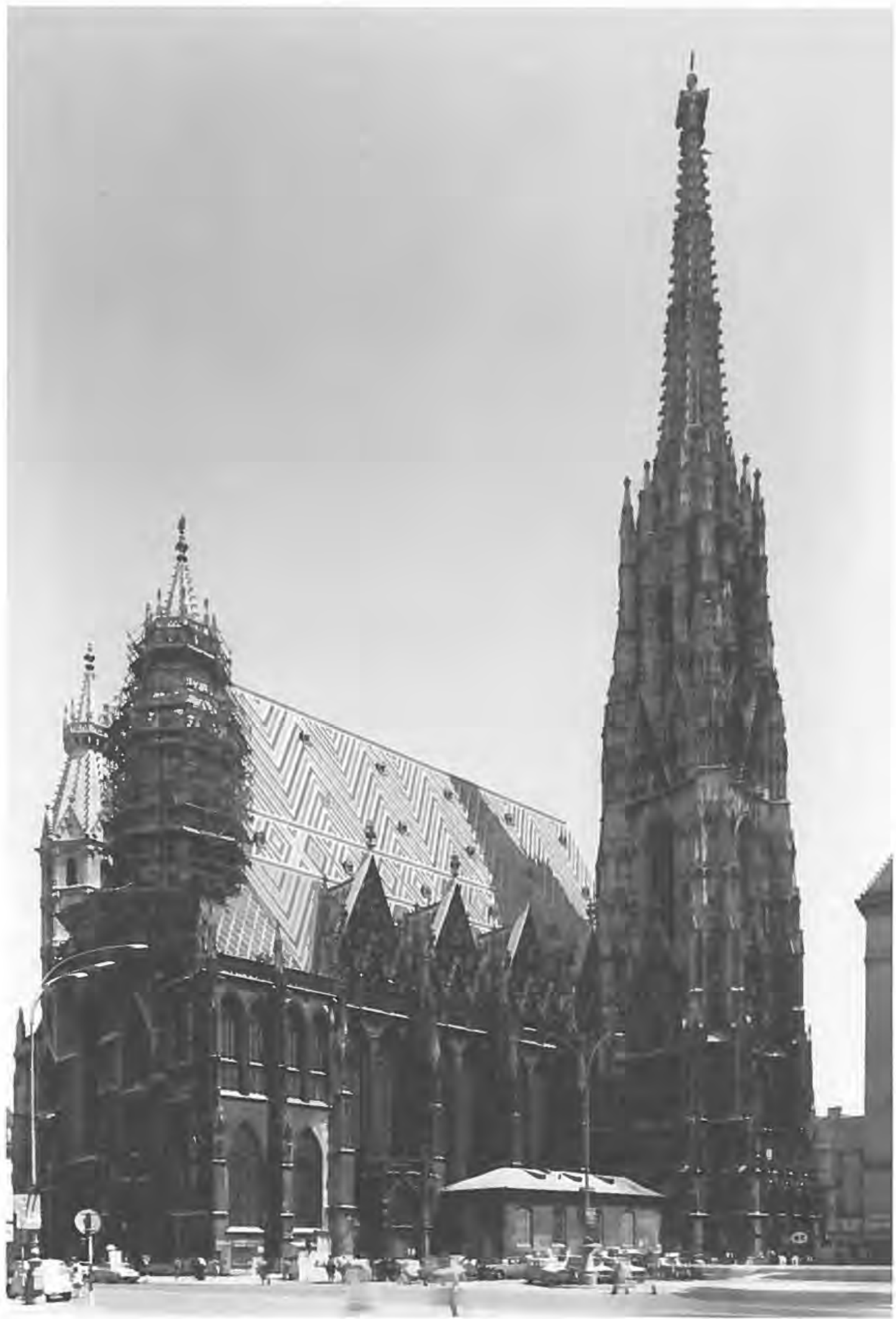


Puchsbaums Nachfolger, der international bekannte Baumeister Laurenz Spenning, beendete den Ausbau des Langhauses, indem er die Einwölbung mit einer dekorativen Rippenfiguration vollendete, und am Außenbau die Giebelgeschosse - vermutlich wohl auch den Friedrichsgiebel - und die Fenstermaßswerke gestaltete. Er plante die westiche Empore neu und dürfte auch zwei Altarbaldachine und den Lettner entworfen haben. Poter Kodera Nordturm von St. Stephan Eotogratie

Das Hauptwerk Laurenz Spennings war aber der Bau des Nordturms, dessen FundamenDach Entwürfen von Laurenz Spenning - mit dem Aufbau des Nordturms.

Laurenz Spenning erlebte noch die Fertigstellung des Turmunterbaues mit der (heutigen) Barbarakapelle, er starb 1477. Seine Nachfolger hielten sich zu einem großen Teil an seine Planvorgaben, als Doppelfenstergeschoss und Glockengeschoss erbaut wurden. Der Turm blieb allerdings unvollendet. 1511 wurden die Bauarbeiten, wohl aus Geldmangel, eingestellt $(A b b .7)$.

Zur selben Zeit bemühte sich Kaiser Friedrich III. (1415-1493) persönlich beim Papst um die Bistumserhebung von Wien, die seit dem Babenberger-Herzog Leopold VI. von den österreichischen Landesfürsten immer wieder forciert wurde. 1469 wurde dafü eine Urkunde ausgestellt und $1480 \mathrm{kam}$ es endlich zu einem feierlichen Akt in der Stephanskirche und der Bekanntmachung der päpstlichen Bullen, indem diese an dem Tor des neuen - unausgebauten - Nordturms angeschlagen wurden.

Nach nunmehr dreihundert Jahren Bauzeit war das von "Stadt* und »Hof* be harrlich verfolgte Ziel erreicht: St. Stephan erhielt - trotz uneinheitlicher Bauplanungas chareristische Eroheinust Sth ond den die einen wichtigen ökonomischen Faktor für die Stadt dargestellt und Generationen der de

literat

UMS-VEREIN 1895-1927

BÖKER Stephansdom 2007

BRAUNEDER/JARITZ Stadtbücher 1989

DEHIO-Handbuch 2007

LOHRMANN/OPLL Regesten 198

OFFENBERGER Untersuchungen 2000

OGESSER Beschreibung 1779
ZYKAN Stephansdom 1981

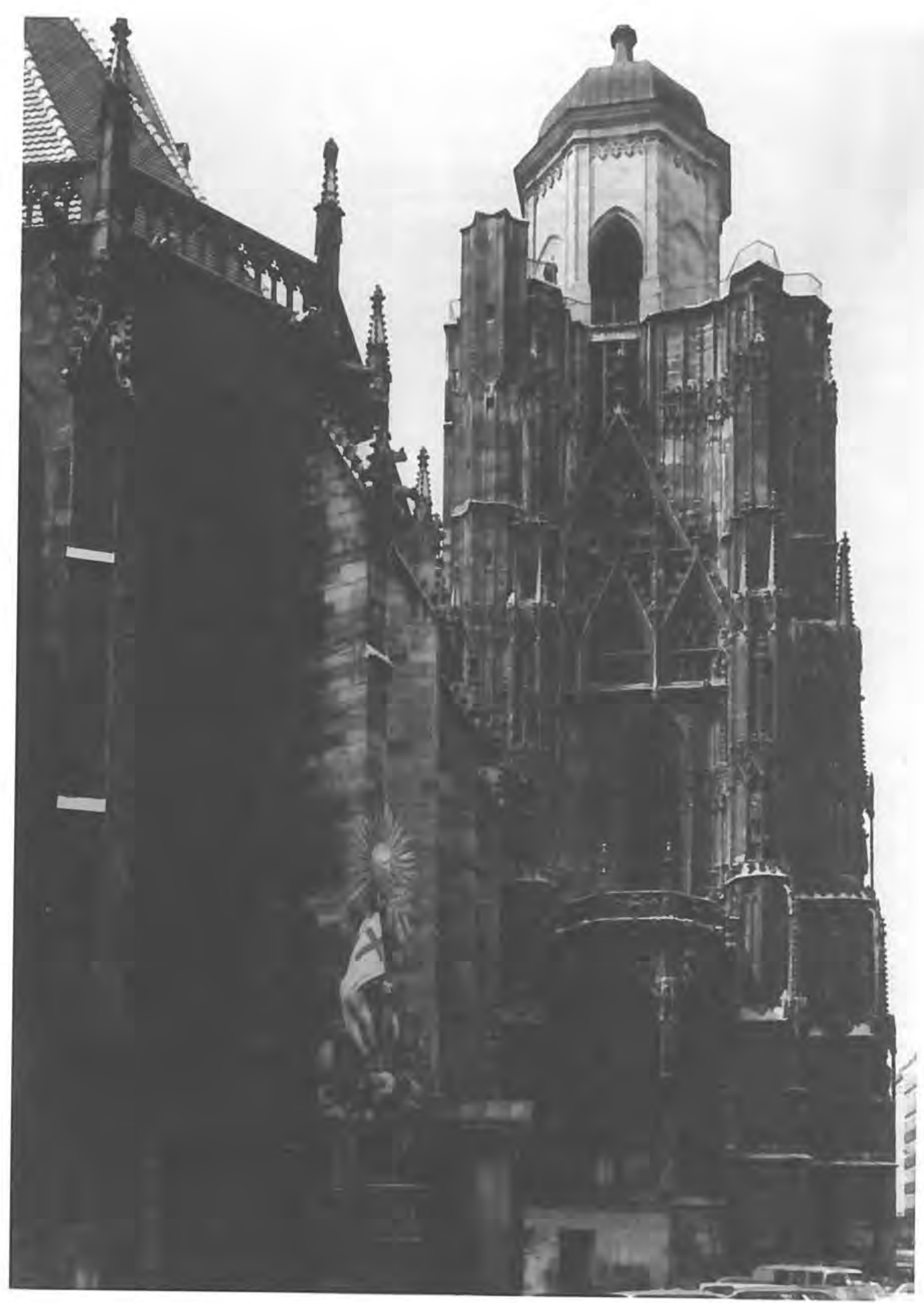


UAA - Universitätsarchiv der Akademie der bildenden Künste

HMW - Historisches Museum der Stadt Wien (heute: Wien Museum)

KuKa - Kupferstichkabinett der Akademie der bildenden Künste Wien

KHM - Kunsthistorisches Museum Wien

ALTERTHUMS-VEREIN 1895-1927

Alterthums-Verein zu Wien ( $\mathrm{Hg}$.): Quelle zur Geschichte der Stadt Wien, Abteilun 1.-111., Wien 1895-1927.

BACHER Glasmalerei 1998

Ernst Bacher: Monumentale Glasmalerei und Museum: Ein Widerspruch?, in: Gesto, Essays on Stained Glass in Memer of Hayward 1918-1994, Bd. 37 (1998) 2 .

S. $135-141$

\section{BACHER Glasmalerei 2004}

Ernst Bacher: Glasmalerei als Bildkuns der mittelalterlichen Architektur. Einig Anmerkungen zu Aspekten des Gesam zusammenhanges, in: Hartmut Scholz Ivo Rauch, Daniel Hess (Hg.): Glas.

Malerei. Forschung. Internationale Studie 2004, S. 23-34.

BECHMANN Villard de Honnecourt 1991 Roland Bechmann: Villard de Honnecourt. La pensée technique ou XIIle siècle et sa communication, Paris 1991.

BECK u.a. Kunst 1975

Herbert Beck u.a.: Kunst um $1400 \mathrm{am}$ Mittelrhein: Ein Teil der Wirklichkeit, Ausstellungskatalog, Frankfurt a.M. 1975

BINDING/LINSCHEID-BURDICH Planen 2002

Günther Binding: Susanne Linscheid. Burdich: Planen und Bauen im frühen und hohen Miterater noch den Sctiriten und bis 1250 , Darmstadt 2002.
BINDING Baubetrieb 1993 Gunther Binding: Boubetrieb im Mittelalter, Darmstadt 1993.

BINDING Bauhütte 1980 Günther Binding: Bouhütte, Lexikon des Mittelalters, Band 1, 1980.

BINDING Bedeutung 2003

Günther Binding: Die Bedeutung von Lich und Farbe für den mittelalterlichen Kirche bau, in: Sitzungsberichte der wissenschaftlichen Gesellschaft an der Johann Wolfgang Goethe-Universität Frankfurt an Main, Band 41 (2003) 3, S. 129-171.

BINDING Gotik 2000

Günther Binding: Was ist Gotik. Eine Analyse der gotischen Kirchen in Frankreich, England und Deutschland 11401350, Darmstadt 2000.

BÖKER Architektur 2005

Johann Josef Böker: Architektur der Gothik Gothic Architecture, Salzburg 2005.

BÖKER Stephansdom 2007

Johann Josef Böker: Der Wiener Stephans dom. Architektur als Sinnbild für das Hous Österreich, Salzburg/Wien/München 2007.

BÖKER Veitsdom 2008

Johann Josef Böker: Veitsdom und Ste. phansdom. Architektonische Beziehungen zwischen karolinischem Prag und rudolphnischem Wien, in: Markéta Jarošová, jiri Kuthan, Stefan Scholz (Hg.): Prag und die großen Kulturzentren Europas in der Zeit der Luxemburger 1310-1437: Internationa Konferenz aus Anlaß des 660 . Jubiläums der Gründung der Karlsuniversität in Prag. Prag 2008, S. 299-312.

BORK U.a. Landschaftsentwicklung 1998 Hens-Budall Bork us: Landschoftsentwicklung in Mitteleuropa. Wirkungen des
Menschen ouf Londschaften, Gotha/Stuttgart 1998.

BORK Türme 2008

Rudolf Bork: Gotische Türme in Mittel-

europa, Petersberg 2008

BORK Great Spires 2003

Rudolf Bork: Great Spires: skyscrapers of the New Jerusalem, Köln 2003.

BRAUNEDER/JARITZ Stadtbücher 198 Wilhelm Brauneder, Gerhard Jaritz ( $\mathrm{Hg}$.): Die Wiener Stadtbücher 1395-1400 (Fontes Rerum Austriacarum, Abt. 3, 10/1), Wien/ Köln 1989.

BRUCKMÜLLER Stephansdom 2005 Ernst Bruckmüller: Stephansdom und Stephansturm, in: Emil Brix, Ernst Bruckmüller, Hannes Stekl (Hg.). Memori Austriae II, Wien 2005, S. 40-74.

BRUNNER Finanzen 192

Otto Brunner: Die Finanzen der Stodt Wien von den Anfängen bis ins 16. Jahrhundert (Studien aus dem Archiv der Stadt Wien Bd.1/2), Wien 1929.

CATHÉDRALES Gothiques 1989 Les bâtisseurs des Cathédrales Gothiques, Ausstellungskatalog, Straßburg 1989.

CHOTẼBOR TUrm 2001

Petr Chotěbor: Der große Turm des St. Veitsdoms: Erkenntnisse, die bei den Instandsetzungsarbeiten im Jahr 2000 gewonnen wurden, Umẻni 49, 2001, S. $262-270$

CONRAD Kirchenbau 1990 Dietrich Conrad: Kirchenbau im Mittel. alter, Leipzig 1990.

DEHIO-Handbuch 2007 DEHIO-Handbuch: (Die Kunstdenk. mäler Österreichs: Topogrophisches
Denkmölerinventar) I. Bezirk - Innere

Stadt, Wien 2007

DIEM Stephansdom

Peter Diem, Der Stephansdom und seine

politische Symbolik, http://peter-diem.at/ Buchtexte/stephansdom.htm

FABER/GRÖNING Daguerreotypie 2005 Monika Faber, Maren Gröning (Hg.) Inkunabeln einer neuen Zeit. Pioniere de Daguerreotypie in Österreich 1839-1850 Wien 2005.

FILLITZ Bildende Kunst 1998

Hermann Fillitz (Hg.): Geschichte der Bildenden Kunst in Österreich Band Früh-und Hochmitter Mand New York 1998.

FISCHER Kirchenbaukunst 1962 Friedhelm Wilhelm Fischer: Die spätgotische Kirchenboukunst am Mittelrhe 1410-1520, an charakteristischen Beispielen dargestellt, nach Schulen geordnet und mit historisch-topographischen Darlegungen verknüpft, Heidelberg 1962

FLIEDER Stephansdom 1968

Viktor Flieder: Stephonsdom und Wiener Bistumsgründung, Wien 1968.

FLUM Baugeschichte 2001

Thomas Flum: Zur Baugeschichte des

Freiburger Münsterturms, Umęni 49, 2001 S. 256-261.

FRIEDERICH Steinbearbeitung 1932 Karl Friederich: Die Steinbearbeitung in ihrer Entwicklung vom 11. bis zum 18. Jahrhundert, Augsburg 1932.

FRODL 19. Jahrhundert 2002 Gerbert Frodl (Hg.): 19. Jahrhundert (Geschichte der bildenden Kunst in Österre Bd. 5), Wien/München 2002.
FRODL-KRAFT Glasgemälde 1962

Eva Frodl-Kraft: Die mittelalterlichen Glas gemälde in Wien (Corpus Vitrearum Med evi), Österreich Bd. 1, Wien/Graz/Köln/ Wien 1962, S. 146-150.

RODL-KRAFT Glasmalerei 1970

Eva Frodl-Kraft: Die Glosmalerei. Entwicklung. Technik. Eigenort, Wien/München

\section{UCHSBERGER Reissboden 1989} Hermann Fuchsberger: Der Reissboden als lechnische Grundloge der mittelalterlichen Boukunst, ungedr. Dipl.-Arbeit, Salzbur

FUHRMANN Beschreibung 1767 Pater Mathias Fuhrmann: Historische Beschreibung und kurz gefaßte Nachrich von der Römisch. Koiserl. und Königlichen Residenz-Stadt Wien und Ihren Vorstädten, 2. Theil, 2. Band, Wien 1767.

GUTDEUTSCH Erdbeben 198 Rolf Gutdeutsch, Christa Hammerl, Ingeborg Mayer, Karl Vocelka, Erdbeben als historisches Ereignis: die Rekonstruktion des Bebens von 1590 in Niederösterreich, Berlin 1987

\section{HECHT Maß 1979}

Konrad Hecht: MOB und Zohl in der gotichen Boukunst, Hildesheim 1979.

JONES Ironwork 200

Richard A. Jones: Ironwork at the Top: a medieval jigsaw puzzle in Salisbury Cathedral's spire, in: Robert Bork (Hg.): De Re Metollica: The uses of metol in the Middle Ages, Aldershot 2005, S. 279-295.

JUCKES Prague - Vienna - Košice 2006 Tim Juckes: Prague - Vienna - Košice: The Church of St. Elizabeth in Košice and Voult Design in the Genertion ofter Peter Parter, in: Zoe Opačić (ed.): Art and Architecture of Medieval Progue and Bohemia. Proceedings of the BAA Annual Conference in Prague 2006, Leeds 2009, S. 117-132.

KASSAL-MIKULA St. Stephan 1997 Renata Kassal-Mikula: St. Stephon im 19. Jahrhundert - Praxis und Ästhetik, in: KATALOG "850 Jahre St. Stephanu 1997 S. 400-407.

KASSAL-MIKULA Schausammlung 1984 Renata Kassal-Mikula in: Robert Waissenberger (Hg.): Schousammlung Historisches Museum der Stadt Wien, Wien, 1984.

KASSAL Alt-Wien 2004

Renata Kassal-Mikula: Alt-Wien unter dem Demolierungskrampen. Wiens innenstadt noch 1858, in: KATALOG Alt-Wien 2004, S. 46-61, 378-399.

KATALOG "850 Jahre St. Stephanu 1997 850 Jahre St. Stephon. Symbol und Mitte in Wien 1147-1997, Katalog zur 226. Sondeausstellung des Historischen Museum der Stadt Wien, Dom- und Metropolitankapite Wien, 24. April bis 31. August 1997, Wien 1997

KATALOG "Alt-Wien“ 2004 Wolfgang Kos, Christian Rapp (Hg.), AltWien Die Stodt die niemols wor Kot

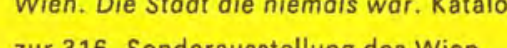
Museums, 25. Nov. 2004 - 28. März 2005 Wien 2005.

KATALOG "Strasbourg 1400" 200 Strasbourg 1400: un foyer d'art dans l'Europe Gothique, Ausstellungskatalog, Straßburg 2008.

KEMP Sermo Corporeus 1987 Wolfgang Kemp: Sermo Corporeus. Die Erzählung der mittelalterlichen Glasfenster. München 1987. 
KIESLINGER/MEJCHAR Steine 1972 Alois Kieslinger, Elfriede Mejchar: Die Stein der Wiener Ringstraße, Wiesbaden 1972

\section{KIESLINGER Steine 1949}

Alois Kieslinger: Die Steine von St. Stephon, Wien 1949.

KLEINDIENST Restauration 188

Franz X. Kleindienst: Die Restauration des St. Stephansdomes in Wien in den Jahren 1853-1880, in: Wiener Dombauvereins-Blat 4 (1884) 27, S. 98-103, 107-108, 111-113.

KLETZL Dombaumeister 1934 Otto Kletzl: Zur Identität der Dombaumeister Wenzel Parler $d . \ddot{A}$. und Wenze von Wien. Wiener Jahrbuch für Kunste. schichte IX (1934) S. 43-62.

KLETZL Kreßberger Fragmente 194 Otto Kletzl: Die Kreßberger Frogmente. Zwei Werkrisse deutscher Hüttengotik. Studie zur Baugeschichte der Westfas. sade des Strabburger Münsters und des Langhauses vom Stephansdom zu Wien. langhous wisse schaftlichen Instituts der Elsaß-Lothringe im Reich (Stuttgart 1941) S. 29.

KLOTZ Baukunst 1965/66

Heinrich Klotz: Deutsche und italienische Boukunst im Trecento, Mitteilungen des Kunsthistorischen Institutes in Florenz 1965/66, S. 171-206.

KOCH Baubetrieb 2002

Rudolf Koch: Baubetrieb und Boutech. nik, in: Ausstellungskatalog Gotikschätz Oberössterreich, Linz 2002, S. 222-234.

KOEPF Planrisse 197 Hans Koepf: Die gotische Planrisse der Ulmer Sammlungen, Ulm 1977.

KOS/RAPP Alt-Wien 2004 Wolfgang Kos, Christian Rapp (Hg.), Alt-
Wien. Die Stadt die niemals war. Katalog zur 316. Sonderausstellung des Wien Museums, 25. Nov. 2004 - 28. März 2005, Wien 2005

KUBA-HAUK/SALIGER Dom- und Diözesanmuseum 1987

Waltraut Kuba-Hauk, Arthur Saliger: Dom und Diözesanmuseum Wien, Erzbischötliches Dom- und Diözesanmuseum ( $\mathrm{Hg}$. Schriftenreihe des Erzbischöflichen Domund Diözesanmuseums Wien, N. F., Bd. 10 (1987) S. 290-292.

LANDESINNUNG Bau Wien 2001 Landesinnung Bau Wien der Wirtschafts. kammer $(\mathrm{Hg}$.), Bauen in Wien, Eine Festschrift der Landesinnung Bau Wien, Wien 2001

LEISCHING Werkstreit 1988

Peter Leisching. Werkstreit zu St. Stepho in Wien in den Jahren 1511-1513, in: Oswin Martinek, Gustav Wächter ( $\mathrm{Hg}$. Arbeitsleben und Rechtsordnung. Festschrift Gerhard Schnorr zum 65. Geburts tag, Wien 1988, S. 805-820.

LIESS Riß 81986

Reinhard Liess: Der Riß B der Straßburger Münsterfossade: eine baugeschichtliche Revision, in: Günter Brucher und Wolfgang T. Müller ( $\mathrm{Hg})$ : Orient und Okziden 5 . Megl Franz, Graz 1986, S. 171-202.

LOHRMANN/OPLL Regesten 1981

Klaus Lohrmann, Ferdinand Opll: Regeste zur Frühgeschichte von Wien (Forschunge und Beiträge zur Wiener Stadtgeschichte 10), Wien 1981

LOOS Innenraum 1906

Adolf Loos: Der schönste Innenraum, der schönste palast, das schönste sterbende gebäude, des schönste neve geburde dor schönste spaziergang in wien. Beantwor. tung einer rundfrage (1906), in: Ders.: Trotzdem. Gesammelte Schriften 1900-1930, Innsbruck 1931, S. 62Julian M. Luxford: In Dreams: The Sculpturallconography of the West Front of Bath Abbey Reasessed, Religion and the Arts 4 2000, S. 314-336.

MÜLLER/ROHATSCH Gesteinsbestand 1993 Harald W. Müller, Andreas Rohatsch u.a.: Gesteinsbestand in der Bousubstanz der Westfassade und des Albertinischen Chores. Österr. Zeitschrift für Kunst und Denkmalpflege, (1993) 3/4, S. 106-11

MÜLLER Grundlagen 1990

Werner Müller: Grundlagen gotischer Bautechnik, München 1990.

OBERHAIDACHER-HERZIG Glasmalere

Elisabeth Oberhaidacher-Herzig: Glasmalerei: Besonderheiten - Auftraggeber - Werkstätten, in: Günter Brucher $(\mathrm{Hg}$. Geschichte der Bildenden Kunst in Österreich. Gotik, 8d. 2, München u.a. 2000 S. 411-432.

OFFENBERGER Untersuchungen 2008 Johann Offenberger: Bouarchäologische Untersuchungen im Bereich der Westonlo. geven St. Stephon in Wien, in: Friedrich

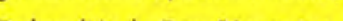
Dahm (Hg) Dos Rient Archologe. che schatten (Verollentlichungen der Kommission für Kunstgeschichte/Österreichisch Akademie der Wissenschaften 8, D Wiener Stephansdom 1) Wien 2008 s. $31-48$.

OGESSER Beschreibung 1779

Joseph Ogesser: Beschreibung der Metropolitankirche zu St. Stephan. Herausgegeben von einem Priester der erzbischöflichen Kur, Wien 1779.
OPLL Leben 1998

erdinand Opll: Leben im mittelalterliche

Wien, Wien/Köln/Weimar 199

OPLL Nachrichten 1995

Ferdinand Opll: Nochinhten aus dem

mittelalterlichen Wien. Zeitgenossen

berichten, Wien/Köln/Weimar 1995.

ORTNER Marquard Hergott 1972 Josef Peter Ortner: Marquard Herrgol (1694-1762), Wien 1972 .

PARUCKI Minoritenkirche 1995 Maria Parucki: Die Wiener Minoriten-

kirche, Wien/Köln/Weimar 1995.

PERGER/BRAUNEIS Kirchen 1977 Richard Perger, Walther 8rauneis: Die mittelalterlichen Kirchen und Klöster Wiens (Wiener Geschichtsbücher 19/20) Wien/ Hamburg 1977.

PERGER Baumeister 1970

ichard Perger: Die Boumeister des Wien Stephansdomes im Spätmittelalter. Wien Jahrbuch für Kunstgeschichte, XXIII

(1970), S. 66-107.

PERGER Ratsbürger 1988

Richard Perger: Die Wiener Ratsbürger

1396-1526, in: Forschungen und Beiträ

(396- Wient

PERGER St. Stephan 1997

ichard Perger: St. Stephan und die Wiener vom 12. bis zum 19. Jahrhundert, in: Katalog "850 Jahre St. Stephanu 1997, S. 36-54.

RÖDER Türme 2003

Bernd Herbert Roder: Turme und Fassaden

von Laon bis Reims, Trier 2003.

ROHATSCH Gesteine 2008

Andreas Rohatsch: Die Gesteine in

der Bausubstanz des Riesentores von

St. Stephan - Gesteinskundlich
Charakterisierung und technische Eigenschaften, in: Friedrich Dahm ( $\mathrm{Hg}$.): Das Riesentor - Archöologie, Bau- und Kunstge IVeröfentlichungen der Kommission r Kunstersion re wisen (umie der Wissenschaten 8, Der Wiener

ROHATSCH Gesteinskunde 1997

Andreas Rohatsch: Gesteinskunde in der Denkmalpflege unter besonderer Berücksichtigung der jungtertiören Naturwerksteine von Wien, Niederösterreich und dem Burgenland. Habilitationsschrift BOKU Wien, Wien 1997

ROHATSCH Passionsreliefs 2009 Andreas Rohatsch. Die Passionsreliets von St Stephan - ein hedeutendes Verwendung seispiel für den Breitenbrunner Bildhouerthin in: A. Husslein-Arco, V. Pirker-Aurenhammer (Hg.): Die Passionsreliets vom Wiener Stephansdom. - Gefä vert - Prösentiert, Wien 2009, S. 93-95.

OOMER-BÜCHNER Wahl- und Krönungskirche 1857

Benedict Jacob Römer-Büchner: Die Wahund Krönungskirche der deutschen Koiser 2u St. Bartholomäi in Erankfurt am Main, Frankfurt a. M. 1857

\section{ORICZER Büchlein 1468}

Matthäus Roriczer: Das Büchlein von der len Gerechtigkeit, Regensburg 1486

G. v. F. Geldner, Wiesbaden 1965

SANDRON L'art 2000 Dany Sandron: " $L$ 'art n'a jamais rien produit de plus élevéu: I'octogone et lo lèche, in: Strosbourg 1400, 2008, s. $100-117$.

SANDRON Les flèches 2009 Dany Sandron: Les flèches de Strasbourg. Ulm, Vienne et Francfort: L'intervention municiplale dans les grands chantiers d'église vers 1400. Revue de l'art 166 . 2009, S. 27-42.

SASS Turm 2000

Manfred SaB: Der kühnste Turm der Chris. tenheit: Eine statisch-konstruktive Glanzleistung des Mittelalters, Münsterblatt: Jahresschrift des Freiburger Münsterbauvereins e.V. 7, 2000, S. 15-22.

SAUVÉ cathédrale de Strasbourg 2010 Jean-Sébastian Sauvé: L apport du dessin d'architecture dans la chronologie de la tour de la cathédrale de Strasbourg. Bulletin de la Cathédrale de Strasbourg 29 2010, S. 9-44.

\section{SCHAFFER Führer 1908}

Franz Xaver Schaffer: Geologischer Führe für Exkursionen im Inneralpinen Wienerbecken II. Teil, Berlin 1908.

SCHOCK-WERNER Straßburger Münster 1983

Barbara Schock-Werner: Das Straßburge Münster im 15. Jahrhundert: Stilistische Entwicklung und Hüttenorganisation eines Bürger-Doms, Phil. Diss., Köln 1983.

SCHREINER Frömmigkeit 2002 Klaus Schreiner ( $\mathrm{Hg}$.): Frömmigkeit im Mittelalter. Politisch-soziale Kontexte, visuelle Praxis, körperliche Ausdrucksformen. München 2002.

SCHREINER Maria 1994 Klaus Schreiner: Maria: Jungfrau, Mutter Herrscherin, Wien 1994

SCHWARZ Altargucker 2002 Michael Viktor Schwarz: Altargucker und Predigtlouscher. Anton Pilgrams Selbstbildnisse in St. Stephan in Wien, in: Ders. Visuelle Medien im christlichen Kult. Follstudien aus dem 13. bis 16. Jahrhundert, Wien/Köln/Weimar 2002. S. 217-250. 
SCHWARZ Giottus Pictor 2, 2008 Michael Viktor Schwarz: Giottos Werke (Giottus Pictor 2), Wien 200B.

\section{SCHWARZ Kathedralen 2005}

Michael Viktor Schwarz: Kathedrale

verstehen ISt. Veit in Prag als röum

lich organisiertes Medienensemble), in:

Elisabeth Varvra (Hg): Virtuelle Räume:

Roumwahrnehmung und Raumvorstellu

im Mittelalter, Berlin 2005, S. 47-68.

SCHWEIGERT Gotische Plastik 2000

Horst Schweigert: Gotische Plastik unter

den frühen Habsburgern von ca. 1280 bis

1358, in: Günter Brucher $(\mathrm{Hg}$.): Geschichte der Bildenden Kunst in Österreich Band 2. Gotik, Wien/New York 2000.

SEELIGER-ZEISS Lorenz Lechler 1967 Anneliese Seeliger-Zeiss: Lorenz Lechler von Heidelberg und sein Umkreis, Heide berg 1967

SHELBY Gothic design 197

R. Lon Shelby: Gothic design techniques. The 15th-century design booklets of $\mathrm{Ma}$ thes Roriczer and Honns Schmuttermay Carbondale a Edwardsville 1977

SONNLECHNER Bürger 2010

Christoph Sonnlechner: Bürger und Wald. Überlegungen zur Nutzung von Wiener Bürgerspitalswäldern im Mittelolter, in: ahrbuch des Vereins für Geschichte der Stadt Wien, Bd. 64 (2010), S. 82-114.

\section{TIETZE Geschichte 1931}

Hans Tietze: Geschichte und Beschreibung

des St. Stephansdomes in Wien (Oster-

reichische Kunsttopographie, Bd. XXIII).

Wien 1931.

TILMEZ Denckwürdigkeiten 1722

Friedrich Tilmez: Außerlesene Denckwür-

digkeiten von der Sowohl Uralten als Kunst.

reichen St. Stephons Dom-Kirchen und

Thürmen zu Wienn in Österreich, 1722

\section{Leihgeber}

TROST Umbau 1843

Die Texte für den Katalogteil wurden von Michaela Kronberger und Barbara Schedl verfasst. Beiträge anderer Autorinnen und Allgemeine Bauzeitung 8 (1843), S. 5-18, Tafel XDII-XDIII.

TSCHISCHKA St. Stephans Dom 1832 Franz Tschischka: Der St. Stephans Dor Wien und seine alten Kunstdenkmale. Wien 1832

UHLIRZ Rechnungen 1902

Karl Uhlirz: Die Rechnungen des Kirchenmeisteramtes von St. Stephan zu Wien, Wien 1902

WIBIRAL/KASSAL-MIKULA Heinrich von Ferstel 1974

Norbert wibiral: Renate Kassal Mikula

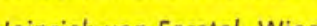

ZISKA Metropolitankirche 1823 Franz Ziska: Die Metropolitankirche zu St. Stephan in Wien.

\section{ZYKAN Stephansdom 198}

Marlene Zykan: Der Stephansdom (Wiener Geschichtsbücher 26/27, Hg. Peter Pötscher), Wien/Hamburg 1981.

ZYKAN Baugeschichte 1970

Marlene Zykan: Zur Baugeschichte des Hochturmes von St. Stephan, Wiener lahrbuch für Kunstgeschichte 23 (1970) S. 28-65.

Andreas Nierhaus

Andreas Rohatsch

B. P.

Beatrix Patzak

c. s.

Christoph Sonnlechner

E.-M. O.

Eva-Maria Orosz

Franz Zehetner

ner

BDA Bundesdenkmalamt, Restaurierwerkstätten Baudenkmalpflege Kartause Mauerbach

Diözesanarchiv Wien

Dï̈zesanmuseum Wien

Dombausekretariat St. Stephan, Wien

Institut für Geotechnik, FB-Ingenieurgeologie, Tu Wien

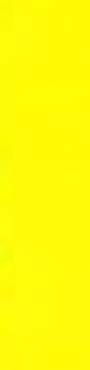

